\title{
ARTIFICIAL INTELLIGENCE AS COGNITIVE AND SOCIOCULTURAL PHENOMENON $^{1}$
}

\author{
Sergey A. Khrapov, Tinatin D. Lopatinskaya,Alexey M. Kashkarov \\ Astrakhan State University, 20a, Tatishchev St., Astrakhan, 414056, Russia. \\ The article is financially supported by the RFBR, project No \\ 18-011-00095 A titled "Technogenic Human: cognitive, sociocultural \\ and virtual aspect of forming".
}

\begin{abstract}
This research is dedicated to the problem of artificial intelligence as a cognitive and sociocultural phenomenon. Subject of the research is detailed in the following parts of the paper: "Extreme position in research and ontologization of artificial intelligence", "Mild position in research and ontologization of artificial intelligence", "Anthropological and sociocultural consequences of artificial intelligence idealization". The main conclusion that the authors made states a special onthological (phenomenological) status of artificial intelligence as a techno-phenomenon, as well as anthropological and sociocultural consequences of its ontologization. In conclusion, the authors define the limits of formation and ontologization of artificial intelligence as a techno-phenomenon.
\end{abstract}

Keywords: artificial intelligence (AI), natural intelligence, consciousness, society, culture, physicalism, functionalism, technologization of human, cognitive security, sociocultural consequences.

\section{INTRODUCTION}

The issue of current state of methodology of artificial intelligence is chosen as a central topic that is analyzed through a lens of classical physicalistic, functionalist and T. Moody, S. Bringsjord, J. Searle, A. Church, G.F. Luger, E.L. Post)[1;2]. The contemporary approaches (D. Dennett, O. Flanagan, T. Polger, N. Block, S. Kripke)[3].The concept of "artificial intelligence" was first introduced into scientific discourse at the International Conference in Washington, D.C. in 1969. We think artificial intelligence can be generally defined as an automatic system reproducing certain functions of human-level reasoning. The methodology of artificial intelligence is based on information concept, physicalistic and functionalistic theories of consciousness.

Physicalistic and functionalist concepts of artificial intelligence reveal the greatest gap. According to the representatives of physicalism, physical and chemical parameters and the substrate of human brain play a primary part in cognitive and intellectual activities. Consequently, futurological expectations of full ontologization of artificial intelligence can be met only when an artificial biological intellectual system is developed, which seems to be hardly feasible, taking into consideration current state of bioengineering.

Representatives of functionalism hold a contrarian viewpoint, proceeding from the available option of modeling different functional states of different systems, regardless of their inherent features and substrate states. This modeling is based on the process of informational self-organization [4]. Essentially, artificial intelligence is currently discussed by representatives of different interpretations of functionalism, holding both mild and extreme positions, as well as scientists with humanitarian orientation who support the extreme position stating that it is intrinsically impossible to model intellectual processes due to their existential nature. We deem that isolating mild and extreme positions in the research and comprehension of artificial intelligence phenomenon gives a conceptual opportunity to achieve a better understanding of its essence as well as its anthropological and sociocultural consequences.

\section{METHODOLOGY}

The methods of the research can be defined as a comparative composition of phenomenological, cognitive, information, sociocultural and socio-anthropological approaches. Conceptualization of substantive as well as 
structural and functional aspects of forming and ontologization of artificial intelligence is carried out by means of combining the philosophical methodology of research with the categorical framework and methods of psychophysiology, social psychology and social linguistics. The originality of the research consists in carrying out a complex philosophical analysis considering how the problem of artificial intelligence creation is correlated with major processes of human technologization based on modern civilization and sociocultural conditions of human existence.

\section{EXTREME POSITION IN THE RESEARCH OF ARTIFICIAL INTELLIGENCE ONTOLOGIZATION}

Extreme position in the issue of the essence of artificial intelligence as a techno-phenomenon in fact determines its full ontologization. In other words, those who share this position do not see any problems in creating an intellectual system, which is absolutely identical to natural intelligence and manifoldly exceeds it in terms of functional capacity, implementing technical means. Moreover, this artificial intelligence is attributed such anthropological features as mind, imagination and understanding.

In philosophical sense this approach is close to vulgar materialism presented in the works by L. Büchner, etc. that reduced mind and consciousness characteristics to brain biological activity. Similarly, parallels are drawn between intellectual capacity of a human and a machine today. It is clear that worldviewessence of this discussion is rooted in the eternal dispute between idealism and materialism.

D. Dennett, a prominent representative of extreme position on artificial intelligence, asserts that neither artificial intelligence nor a human being possesses original intentionality (i.e. immanent spirituality that singles out a human being among other things). He believes that the myth of original intentionality is one of the most profound prejudices integrated into the classical tradition of Western philosophy. Hence, artificial intelligence is truly similar to human consciousness, though not in having original intentionality like a human-being but in lacking it. It is not artificial intelligence that is similar to a human but it is a human that is similar to artificial intelligence[3].

The mentioned principle is dwelt upon in details by foreign scientists: N. Block's "Inverted Earth" (1990), reanimating the idea set forward in S. Kripke's Land of Doubles" (1972), T. Moody's "Land of Zombies", S. Bringsjord's "Ork's Planet" (2003). According to this approach, zombies are creatures used to perform thought-experiments; their behavior does not have any external differences from people's behavior. Zombies have the same skills of finding places and defining time, drive cars and dine out, speak on any topic, including philosophic ones, take decisions in the Parliament, teach others and make moral actions [5]. These forecasts and 'scientific fantasies' are supported by specific technical achievements in the sphere of artificial intelligence and technologization of a human being, upon the whole.

The D. Dubrovsky contends: "In 2020-2030 immortality will become available for the people living in the developed countries, and 10-20 years later for the main body of the humankind" [4]. All the above studies and projects assume that future artificial intelligence medium (a supercomputer, techno-human, zombie, humanoid etc.) will not have consciousness, self-identity, intentionality, i.e. the most important attributes of ontological status of a human being (N. Block, R. Kirk).

It is obvious that following extreme position presupposes that consciousness and intentionality are insignificant for performance of intellectual activity (D. Dennett, O. Flanagan and T. Polger) that is basically seen in a narrow sense as a set of logically organized intellectual operation. This approach is based on classical Dennett's position asserting the issue of modeling conscious activities by means of technical means is senseless as humans themselves do not, in fact,have these mental conditions. Consequently, all highly intellectual activities can be performed without being apprehended as consciousness is excessive from metaphysical, logical, linguistic and empirical point of view [3]. If we agree with this extreme position, there are no barriers to the development ofartificial intelligence and technologization of a human being!

We believe this approach presents an excessively simplified understanding of the essence of human intellectual activities and a human upon the whole. Moreover, even if we temporarily look beyond the principal worldview paradigms of materialists and idealists and try to approach the problem in a formal and 
logical way it becomes evident that there are a number of serious drawbacks in the argumentation system of extreme position representatives. These drawbacks,casting reasonable doubt on its veracity, are connected not only with phenomenological but also with cognitive reduction that in its turn presupposes significant violation of logical norm of technical modeling, since modeling as the representation of original sample is based on creating an identical copy. It differs from the reductionist method proposed by D. Dennett and his followers, because it, in fact, reduces the original sample to the criteria of the desired copy.

In this context, J. Searle holds an absolutely correct position stating thatno AI, in spite any intensification in the sphere of technology, will be able to achieve the level of human consciousness as it does not have potential to overcome the fundamental gap between original and secondary intentionality[1;6].

\section{II.MILD POSITION IN THE RESEARCH OF ARTIFICIAL INTELLIGENCE ONTOLOGIZATION}

Defining mild position in the interpretation of the prospects of artificial intelligence and technologization of a human, as we see it, is even more important than defining extreme one due to its inconsistency and absurdity. In addition to that, mild position considering AI as a heuristic instrument in the study and improvement of human intelligence and cognition is still more objective than extreme point of view of some religiously and existentially oriented scientists who ultimately deny any possibility of studies in this area.

Representatives of mild position adhere to the idea that development of AI as a tool to optimize humans' activities might be reasonable and positive at large. Careful mild approach to the AI projects gives conceptual opportunity for more critical, differentiating assessment of their feasibility and prospects, as well as anthropological and sociocultural consequences.

Consolidating numerous researches of the representatives of this approach (J. Searle, A. Church, G.F. Luger, D.I. Dubrovsky, P.S. Novikov, A.A. Markov, and E. Post and many others), we can express generalized idea that artificial intelligence, a techno-human will always be secondary to natural intelligence and biological human, not only because the latter takes an agent's position, but because it is essentially impossibleto technically model some human features and characteristics. It is these features and conditions of a human that cannot be modeled that serve as an argument for "mild position" of interpretation and ontologization of artificial intelligence and techno-human.

A.N. Kochergin gives the following arguments to support this position [7]:

1) The existence of algorithmically unsolvable tasks makes it impossible to model creative mental processes. These tasks include recognition of deducibility, identification of group theory, recognition of work equivalency in any numeration.

2) A task solving process is to be formalized if it needs to be modeled, but full formalization is impossible, so there are some task-solving processes that cannot be modeled.

3) Creative functions of reasoning cannot be described employing mathematical tools.

4) Modeling of mental processes is possible only within the framework of deduction, i.e. drawing conclusions on the basis of a bulk of knowledge, but creative process is not limited to deduction.

5) It is possible to model only the task that have already been formulated, but it is impossible to model goal setting and interpretation of the obtained results that pertain to creative activity.

These arguments can hardly be refuted. All of them analyze cognitive aspect of the problem of artificial intelligence and techno-human. Moreover, the complexity of intellectual activity embraces deeper layers of human existence (unconscious motifs, illogical and divergent thinking, self-consciousness etc.), significantly strengthening the general argument ofdistinguishingthose features and processes of human's intellectual activity and his existence that can be modeled and those that cannot. We can fully agree with G. F. Luger 
who pointed out: "Perhaps machine intelligence is simply different from human intelligence and trying to evaluate it in human terms is a fundamental mistake"[2].

\section{ANTHROPOLOGICAL AND SOCIOCULTURAL CONSEQUENCES OF ARTIFICIAL INTELLIGENCE IDEALIZATION}

Having dwelt on the modern methodology of AI as a techno-phenomenon, we shall proceed to the main issue of the paper: the issue of technologization of human. The course of technical development today evidently determines main reference points of socio-anthropogenesis [8;9]. The theme of 'artificial' being far more valuable than natural is actually becoming an ideology of social life[10;11;12].In this civilization context, researches in the field of artificial intelligence and anthoropotechnology, whether the authors adhere to mild or extreme position, raise an ontological issue of finiteness, putting some limit to this line anthropogenesis or eliminating all limits and barriers.Moreover, the latter option a priori presupposes possibility of the end of humans' biological existence and the beginning of their existence as a technological being (a technophenomenon). This problematic also aggravates issues connected with ethical and axiological fundamentals as well as consequences of technogenic (electronic) culture and civilization that are considered in the works by a renowned philosopher, L.V. Baeva[13].

\section{CONCLUSION}

The questions on the limits of artificial intelligence and techologization of a human that we set forth in the paper a requite wide and clearly do not offer unambiguously right answers, however, philosophical analysis of current state of AI methodology (artificial personality, artificial society) allow such to make the following conclusions:

1. There are limits to the technologization of a human, as there is finiteness to any process and transformation of any object (and in this case a human acts as an object).

2. The limits to technologization of a human as a process of structural and content transformation, modeling with technical means employed are defined by the contours of human life (biological, psychological (cognitive), sociocultural and existential).

3. There is modest potentiality of technologization of biological contours of human's life. Numerous researches in the sphere of bioengineering that are enhanced in human's worldview by steadily increasing axiologization of technogenic, body-centrism and transionality can serve as an evidence to it (J. Baudrillard).

4. There is a considerable potential in technical modeling of a number of psychological, cognitive and intellectual processes, for example, information recording and storage, calculations, behavioral activities of mechanical character. There are limits to the reproduction of central nervous system in the area of mental, existential acts (processes of self-comprehension, unconscious, creativity).

5. Social components of sociocultural contours of human life will be massively technologized in the near future - there are no limits to technologization in this aspect. If we look at cultural components that mostly have phenomenal, irrational nature (language, collective and social unconscious, mentality, temporhythm of civilization development, autopoiesis, identity), their technologization is impossible, because modern technical and mathematical methodologies do not have methods to cognize them and consequently to change them.

6. Existential contours of human life are the absolute limits to technologization. It is impossible to reproduce them because they are subject and ideal, phenomenal (transcendent) by nature. No technical means can ever mentally express faith, love, reflection, friendship, empathy, grief, hostility, etc., as they cannot be mathematically modeled, being essentially irrational.

What prospects await human-beings in the future? The answer depends on the choice of 'mild' or 'extreme' trajectory of thought and technical development. Reassuring sociocultural and existential contours (constants) of human life- as we believe - are unchanging and will act as limits to technologization of 
intelligence and human as long as the fact of their existence - and what's more, valuable existence - is acknowledged, otherwise the era of zombies is inevitable...

\section{REFERENCES}

Searle, J., 1988. Intentionality: An essay in the philosophy of mind. Cambridge University Press, pp: 278. - Yaz. Engl.

Luger, G.F., 2003. Intelligence: strategies and methods for solving complex problems, Moscow: Publishing house "William", pp: 864. - Yaz. Rus.

3. Dennett, D., 2005. Sweet dreams: Philosophical obstacles to a science of consciousness. Cambridge University Press, pp: 199. - Yaz. Engl.

Dubrovsky, D.I., 2003. New reality: man and computer. Polygnosis, (3), 20-32. - Yaz. Rus.

Alekseev, A. Y. \&Kuraeva, T.A. \&Tumasyan, A.K., 2005. The problem of zombies and prospects for artificial personality and artificial society projects.New in artificial intelligence.Methodological and theoretical issues. Ed. D. I. Dubrovsky\& V. A. Lectorsky, Moscow: Inintell, pp: 26-32. - Yaz. Rus.

6. Searle, J., 1970. Speech acts: An essay in the philosophy of language.Cambridge University Press, pp: 203. - Yaz. Engl.

Kochergin, A.N. 2005.Artificial Intelligence and Thinking.New in Artificial Intelligence.Methodological and theoretical issues. Ed. D. I. Dubrovsky\& V. A. Lectorsky, Moscow: Inintell, pp: 53 - 56. - Yaz. Rus.

Khrapov, S. A., 2014. Technogenic human: sociocultural ontologization problems. VoprosyFilosofii [Problems of Philosophy], (9), 66-75. - Yaz. Rus.

Kryuchkova, S., Khrapov, S., Mironova, Y., Tyrnova, N., \&Leonova, O., 2017.The Russian Public Consciousness Metamorphoses in Conditions of Technogenic Sociocultural Reality. Journal of History Culture and Art Research, 6(4), 1365-1373. - Yaz. Engl.

Khrapov, S. A. \&Novikov A. S., 2013. Virtual sociality: sociocultural analysis. Historical, philosophical, political and juridical sciences, culturology and art history.Theory and practice issues, 7(2), 189-193.- Yaz. Rus.

Baeva, L.V., 2014. Existential and ethical values in an information era. Journal of Human Values, (1): 3343. - Yaz. Engl.

12. Baeva, L.V., 2014. New challenges for humans in the context of e-culture.International Journal of Technoethics, (1): 59-68. - Yaz. Engl.

Khrapov, S. A. \&Kashkarov, A. M., 2017. Man in Technogenic Society: philosophical and historical analysis. Bulletin of the Kalmyk University, (1), 158-163. - Yaz. Rus.

Baeva, L., 2016. Virtual Communication: Strengthening of Real Relationships or Simulation? International Journal of Technoethics, (7): 51-61. - Yaz. Engl. 